\title{
Overcoming Instrumentation Limitations in Spin Tests Using Flight Path Reconstruction Techniques
}

\author{
Joaquim N. Dias ${ }^{1}$ \\ 1 IPEV - Instituto de Pesquisas e Ensaios em Voo, \\ Praça Mal. Eduardo Gomes, 50, CEP 12.228-901, São José dos Campos, São Paulo, Brazil \\ diasjnd@ipev.cta.br
}

\begin{abstract}
This work presents the results obtained by application of flight path reconstruction (FPR) techniques to flight data collected during spin tests. The FPR methodology allows in-flight calibration of several sensors, because it takes advantage of the kinematical redundancy between parameters that usually exists in most flight test instrumentation (FTI) projects. For the particular case of spin tests, the aircraft experiences high angles of attack and sideslip, which may extrapolate the calibration limits of the vanes. Besides that, the Pitot tubes measurements become unreliable when subjected to extreme incidence angles, which corrupt airspeed measurements during the spin. However, measurements obtained before the spin entry and after spin recovery are still valid. Therefore, using the FPR, airspeed measurements are integrated along the maneuver, in order to match the data before and after spin, so that the airspeed during the spin is obtained. This methodology also provides calibration for airflow angle vanes and the values of biases that affected the accelerometers and gyros. In this work, FPR was applied to spin tests performed with three different aircraft, featuring different FTI projects. Despite the different limitations on each FTI project, the FPR was able to provide good results in all cases analyzed.
\end{abstract}

Key words: spin tests, instrumentation errors, flight path reconstruction, IMU biases, and FTI limitations.

\section{Introduction}

Flight path reconstruction (FPR) is a useful procedure for checking the flight test data, in order to assess and correct instrumentation errors, and is capable of dealing with flight data corrupted by both measurement and process noise [1]. This technique is usually applied to flight test data prior to parameter estimation and is also called data compatibility check [2], because it takes advantage of the kinematic redundancy between parameters that usually exists in most flight test instrumentation (FTI) projects. From FPR, it is possible to obtain: biases in accelerometers and rate gyros, inflight calibration of flow angle vanes and calibration of Pitot-static systems, for example, depending on the measurements available. If, for some reason, any of the FTI parameters becomes unreliable, it is not possible to use it in the FPR. Otherwise, it will provide wrong estimates, because the minimization algorithm would try to match experimental data for which the kinematic relationships do not hold anymore. This is the case, for example, of airspeed measurements using Pitot tube at high angles of attack (AOA). During spin tests, the AOA can easily reach 50 degrees. In this work, a modification in the technique is proposed. Instead of dropping the entire airspeed data, the cost function is modified to ignore the differences between model and experimental data when the angle of attack becomes too high. This approach allows avoiding wrong estimates that arise when trying to match unreliable data, but still makes it possible to use the good parts of the data. As the algorithm tries to match the airspeed before the spin and after recovery, the best estimate for the true airspeed during the spin is naturally obtained by integration between these two parts. Comprehensive examples are presented using data from spin tests performed with three aircraft, featuring different FTI projects. For the first aircraft, the only FTI limitation is the Pitot tube measurements at high AOA, whereas the other two aircraft have additional issues regarding attitude angles during the spin. Using the same approach to all cases, the intervals of valid data were identified for each instrumentation parameter, so that the FPR was able to reconstruct the data along the entire manoeuvre. 


\section{Test Vehicles and Instrumentation}

The first aircraft studied is the Embraer A-29 Super Tucano, depicted in Fig. 1. The instrumentation provided the following parameters, acquired at $32 \mathrm{~Hz}$ : Euler angles $(\phi$, $\theta, \psi)$, body-axes angular rates $(p, q, r)$, linear accelerations $\left(a_{x}, a_{y}, a_{z}\right)$, airspeed, altitude and engine parameters, all collected from data bus. Additionally, an air data boom was installed on the right wing tip, providing angle of attack and sideslip.

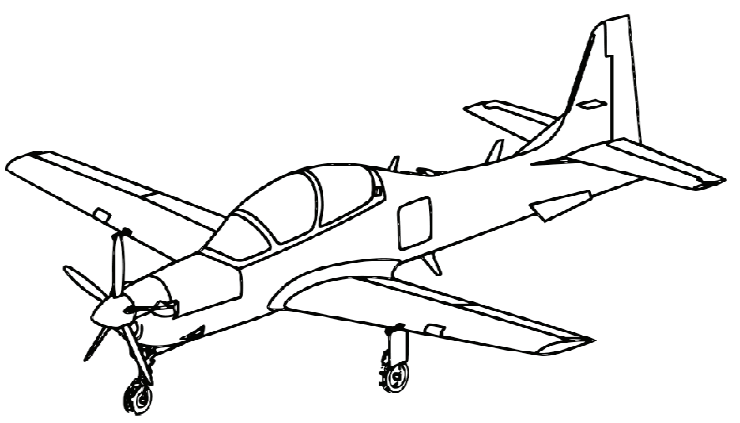

Fig. 1. Embraer A-29 Super Tucano.

The second aircraft presented is the Embraer T-27 Tucano (Fig. 2). The instrumentation project also provided the same parameters as the Super Tucano, but the aircraft does not feature a data bus. Therefore, angular rates and accelerations were collected from rate gyros and accelerometers installed onboard the aircraft. Pitch and roll angles were obtained from a vertical gyro, whereas the heading was derived from aircraft navigation instruments, namely the horizontal situation indicator (HSI), which is basically a compass. Air flow angles were measured by an air data boom on the right wing as well.

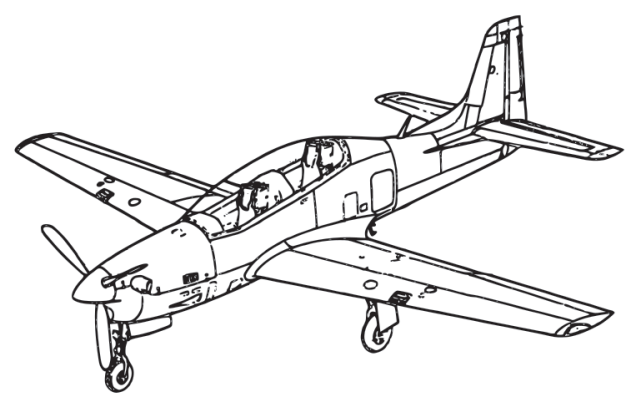

Fig. 2. Embraer T-27 Tucano.

The third aircraft analyzed is the Embraer AT26 Xavante (Fig. 3), which is a jet trainer. The instrumentation available was basically the same as the T-27 Tucano, since this aircraft does not feature data bus. The only difference is that the air data boom is mounted on the nose of the aircraft. The Pitot tubes of the aircraft are positioned at the nose, being susceptible to the aerodynamic wake of the nose when the aircraft is flying at high angles of attack or sideslip. So, the different FTI projects experienced different limitations.

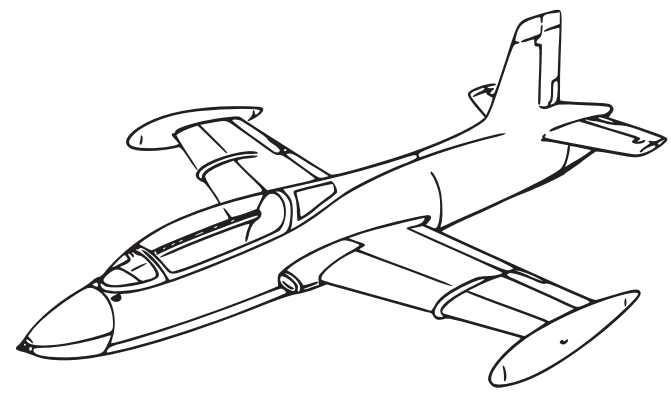

Fig. 3. Embraer AT-26 Xavante.

Flight Path Reconstruction: Formulation

The complete set of kinematical relationships used for flight path reconstruction is given by [1]:

$$
\begin{aligned}
& \dot{u}=-q \cdot w+r \cdot v-g \cdot \sin \theta+a_{x, C G} \\
& \dot{v}=-r \cdot u+p \cdot w+g \cdot \cos \theta \sin \phi+a_{y, C G} \\
& \dot{w}=-p \cdot v+q \cdot u+g \cdot \cos \theta \cos \phi+a_{z, C G} \\
& \dot{\phi}=p+q \cdot \sin \phi \tan \theta+r \cdot \cos \phi \tan \theta \\
& \dot{\theta}=q \cdot \cos \phi-r \cdot \sin \phi \\
& \dot{\psi}=q \cdot \sin \phi \sec \theta+r \cdot \cos \phi \sec \theta \\
& \dot{h}=u \cdot \sin \theta-v \cdot \cos \theta \cdot \sin \phi-w \cdot \cos \theta \cos \phi
\end{aligned}
$$

Ideally, the aircraft states can be obtained by numerical integration using the accelerations and rotation rates measurements. From the states, it is possible to calculate true airspeed and air flow angles:

$$
\begin{gathered}
V=\sqrt{u^{2}+v^{2}+w^{2}} \\
\alpha=\arctan \left(\frac{w}{u}\right) \\
\beta=\arcsin \left(\frac{v}{v}\right)
\end{gathered}
$$

However, simple state integration usually does not agree with measured data, due to small biases in accelerometers and gyrometers measurements. Using a proper error model, 
these biases and other instrumentation errors can be corrected.

The accelerometers and gyrometers measurements are usually corrupted by a constant bias:

$$
\begin{array}{ll}
a_{x}=a_{x m}-b_{a_{x}} & p=p_{m}-b_{p} \\
a_{y}=a_{y m}-b_{a_{y}} & q=q_{m}-b_{q} \\
a_{z}=a_{z m}-b_{a_{z}} & r=r_{m}-b_{r}
\end{array}
$$

The flow angles measured by the vanes are not exactly the true values, but are usually correlated to them by linear relationships:

$$
\alpha_{m}=K_{\alpha} \cdot \alpha+b_{\alpha} \quad \beta_{m}=K_{\beta} \cdot \beta+b_{\beta}
$$

Additionally, the sideslip angle measured by vanes mounted on an air data boom is slightly different from the above definition, and is usually referred to as flank angle:

$$
\beta_{v}=\arctan \left(\frac{v}{u}\right)
$$

The flank and sideslip angles are related by $\tan \beta=\tan \beta_{v} \cdot \cos \alpha$. For low angles of attack, there is virtually no difference between the two definitions above, but for very high angles of attack maneuvers, such as spin, it is important to apply the correct definition to the data collected.

The airspeed is usually measured by an air data boom installed away from the CG position. Consequently, these measurements are affected by aircraft rotation rates $(p, q, r)$ during a spin, but a proper correction can be applied provided the location of the total pressure port relative to the $C G$. The velocity on any point $P$ can be obtained by [3]:

$$
\vec{V}_{P}^{B}=\vec{V}_{C G}^{B}+\vec{\omega} \times \vec{r}_{P}
$$

Where $\vec{\omega}$ is a general angular velocity and $\vec{r}_{P}$ is a general position vector with respect the CG along the body axes, given by:

$$
\vec{\omega} \times \vec{r}_{P}=\left|\begin{array}{ccc}
\hat{i} & \hat{j} & \hat{k} \\
p & q & r \\
x_{P} & y_{P} & z_{P}
\end{array}\right| \begin{array}{r}
\left(q \cdot z_{P}-r \cdot y_{P}\right) \hat{i}+ \\
\left(r \cdot x_{P}-p \cdot z_{P}\right) \hat{j}+ \\
\left(p \cdot y_{P}-q \cdot x_{P}\right) \hat{k}
\end{array}
$$

Considering the point $\mathrm{P}$ as the air data boom location, the $\mathrm{u}, \mathrm{v}$ and $\mathrm{w}$ components at the CG location can be readily obtained:

$$
\begin{aligned}
& u=u_{\text {boom }}-\left(q \cdot z_{\text {boom }}-r \cdot y_{\text {boom }}\right) \\
& v=v_{\text {boom }}-\left(r \cdot x_{\text {boom }}-p \cdot z_{\text {boom }}\right) \\
& w=w_{\text {boom }}-\left(p \cdot y_{\text {boom }}-q \cdot x_{\text {boom }}\right)
\end{aligned}
$$

The model for estimation of instrument errors is given by:

$$
\begin{aligned}
& \dot{x}(t)=f[x(t), u(t), \Theta], x\left(t_{0}\right)=x_{0} \\
& y(t)=g[x(t), \Theta] \\
& z\left(t_{k}\right)=y\left(t_{k}\right)+v\left(t_{k}\right)
\end{aligned}
$$

This model accounts only for measurement noise $v$ on the output variables. The state, input and observation vectors are given by:

$$
\begin{aligned}
& x=\left[\begin{array}{lllllll}
u & v & w & \phi & \theta & \psi & h
\end{array}\right] \\
& u=\left[\begin{array}{lllllll}
a_{x} & a_{y} & a_{z} & p & q & r
\end{array}\right] \\
& y=\left[\begin{array}{lllllll}
v & \alpha & \beta & \phi & \theta & \psi & h
\end{array}\right]
\end{aligned}
$$

The vector $\Theta$ of unknown parameters to be estimated include not only the instrumentation errors, but also the initial conditions of the states:

$$
\begin{aligned}
& \Theta=\left[\begin{array}{lllllll}
b_{a_{x}} & b_{a_{y}} & b_{a_{z}} & b_{p} & b_{q} & b_{r} & \cdots
\end{array}\right. \\
& \begin{array}{llllll}
K_{\alpha} & b_{\alpha} & K_{\beta} & b_{\beta} & \ldots
\end{array} \\
& \left.\begin{array}{lllllll}
u_{0} & v_{0} & w_{0} & \phi_{0} & \theta_{0} & \psi_{0} & h_{0}
\end{array}\right]
\end{aligned}
$$

As the instrumentation was located very close to the CG, no corrections were applied to accelerations due to off-CG location. Besides that, no time delays were considered in the error model. 
Besides these common instrumentation errors used in classic flight path reconstruction approach, there were additional errors related to spin tests and problems inherent to the FTI used in this work:

- Airspeed measurements obtained using the air data boom suffered from stochastic perturbations due to angles of attack in excess of $40^{\circ}$, but it was not possible to simply filter the data, because there was also a deterministic content that represents the actual variation of true airspeed during the spin. This error affected all aircraft tested;

- Heading measurements were derived from aircraft horizontal situation indicator (HSI), which causes considerable delays. This error affected the T-27 and the AT-26, whereas the A29 featured a magnetometer;

- On the T-27 and AT-26, pitch angle measurements were restricted to +/$85^{\circ}$, because of the mechanical limit of the vertical gyro. If these aircraft eventually reach $\theta=-90^{\circ}$ during the spin maneuver, there will be a "gimbal lock". From this point on, all theta measurements are affected by a nonconstant offset. As roll angle measurements are obtained from the same gyro, these values are also affected after the aircraft reaches $\theta=-90^{\circ}$;

- Altitude measurements were based on static pressure values, which are affected by large sideslip angles during the spin. This error affected all three aircraft.

Overall results: airspeed, air flow angles, Euler angles, IMU (accelerations and rotation rates) and baro altitude measurements are eventually corrupted. Fortunately, not all parameters are invalid all the time. In order to apply a suitable error model, it is necessary to select appropriate intervals of the data, so that the intervals considered useless are not taken into account by the cost function. This is necessary because the simple error model described earlier does not take into account several aspects that affected the collected data, e.g.:

- The effect of high angle of attack and sideslip on Pitot measurements;

- The dynamics of the aircraft HSI during high yaw rates;
- The offset that occurs in the gyro measurements after the aircraft reaches $\theta=-90^{\circ}$ (pitch down).

Therefore, it is not possible to match the observation vectors during the entire maneuver. It is necessary to exclude some portions where the data became unreliable, otherwise the estimation algorithm will try to minimize the overall error, leading to a not matching condition even in the low angle of attack sections of data.

\section{Flight Path Reconstruction: Results}

In order to allow proper estimation of the biases about all three axes, the flight path reconstruction was applied to concatenated maneuvers: a sequence of doublet inputs in all axis and the spin maneuver. The air flow angle vanes calibration coefficients $\left(K_{\alpha}, b_{\alpha}, K_{\beta}, b_{\beta}\right)$ were considered the same for all maneuvers. The six IMU biases $\left(b_{a_{x}}, b_{a_{y}}, b_{a_{z}}, b_{p}, b_{q}, b_{r}\right)$ were allowed to be different for each maneuver, as were the initial conditions $\left(u_{0}, v_{0}, w_{0}, \phi_{0}, \theta_{0}, \psi_{0}, h_{0}\right)$, totalizing 30 parameters to be estimated simultaneously.

The following plots show the comparison between flight test data and parameters reconstructed, and the intervals of invalid data selected for each aircraft.

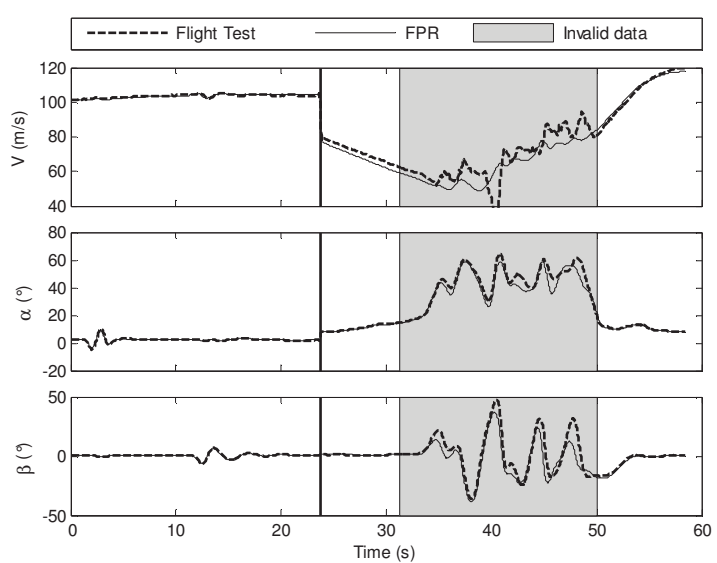

Fig. 4: FPR results for airspeed and flow angles (A29 Super Tucano).

From Fig. 4, it can be seen that the airspeed measurements suffer stochastic variations, but there is also a deterministic content, represented by the reconstructed values. The same invalid data interval for airspeed was 
considered for flow angles. This was necessary because the simple linear relationship between true and vane angles cannot ensure proper modeling of the dynamics of vanes during the spin. For low angles of attack and sideslip, it can be seen that the error model matches the flight data quite well, before and after the spin. Moreover, the fact that a continuous integration was performed along the entire spin maneuver indicates that the FPR data during the invalid range is somewhat reliable. The same analysis is applicable to the other aircraft. From Fig. 5, it can be seen that the Euler angles measured by the FTI during the spin maneuver are fairly close to the reconstructed values, indicating that they are correct. The values of altitude are slightly different than the measured ones, probably due to errors on the static ports during the spin.

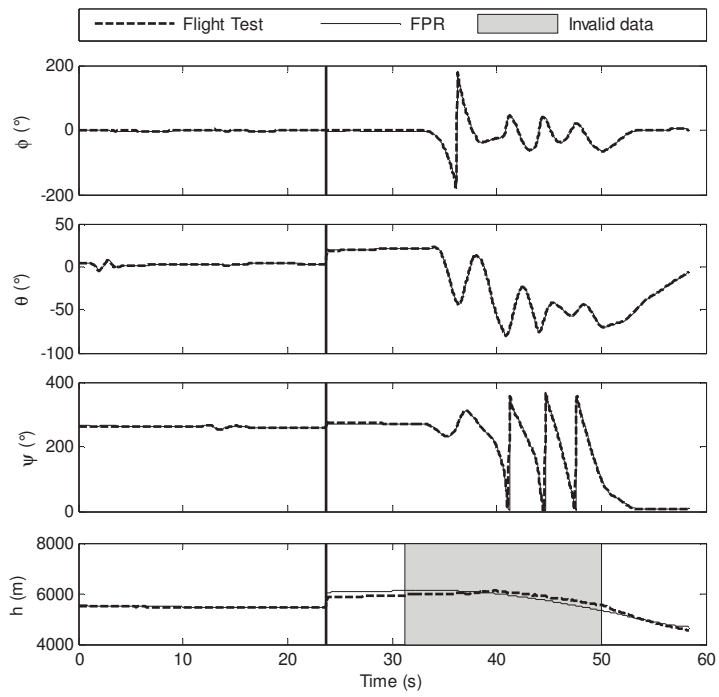

Fig. 5: FPR results for Euler angles and altitude ( $A$ 29 Super Tucano).

The results for the T-27 Tucano are shown in Fig. 6 and Fig. 7. The invalid ranges for Euler angles in Fig. 7 were selected based on limitations of the instrumentation. The $\phi$ and $\theta$ angles were considered unreliable after the aircraft reaches $\theta=-90^{\circ}$ for the first time during the spin. The heading measurements were unreliable when rapid variations occurs, due to the slow response of aircraft HSI. It is noteworthy that now the heading values during the spin make more sense, as it varies $360^{\circ}$ at each turn. After the recovery, when the gyration stops, FPR and measurements for heading converge slowly. For $\phi$ and $\theta$, it is interesting to check the values during recovery. Significant offsets might occur, with the FPR values making more sense.

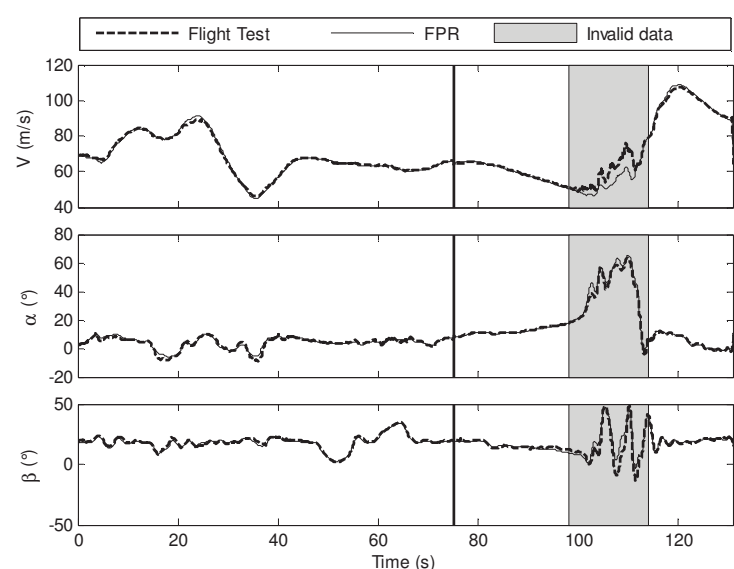

Fig. 6: FPR results for airspeed and flow angles ( $T$ 27 Tucano).

It is important to note that, during the two complete rolls executed initially, the heading measurements provided by the compass were invalid and disregarded accordingly.

The altitude for the T-27 was affected only by small amplitude noise due to large sideslips during spin. So, the invalid range considered was the same as that for airspeed and flow angles.

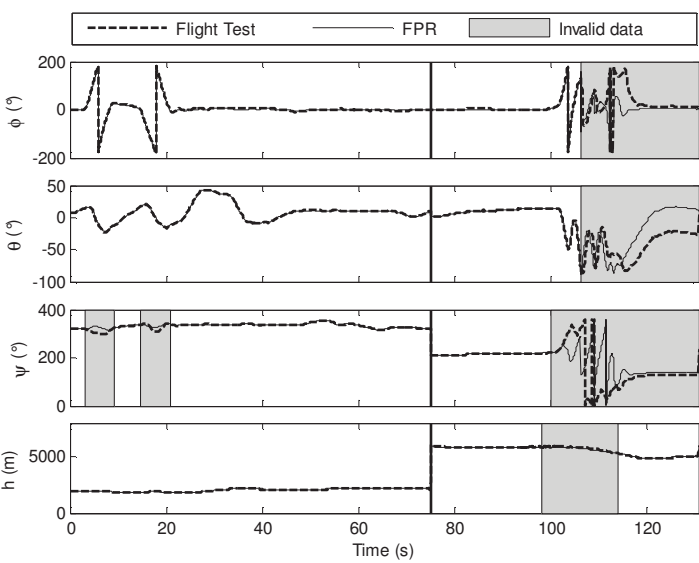

Fig. 7: FPR results for Euler angles and altitude ( $T$ 27 Tucano).

The results for the AT-26 Xavante are shown in Fig. 8 and Fig. 9. During the spin entry, when the aircraft executes two complete rolls, the airspeed measured dropped to almost zero, indicating severe errors on Pitot measurements. There are also significant errors during the second maneuver on these charts. This maneuver is the response to a rudder doublet, when the aircraft oscillates in dutch roll mode. Because of the large sideslip angles, the Pitot 
tube in the nose is affected by the wake caused by the aircraft nose. Besides that, the rapid yaw oscillations cause delay on the aircraft compass, making it necessary to ignore these points for the heading measurements.
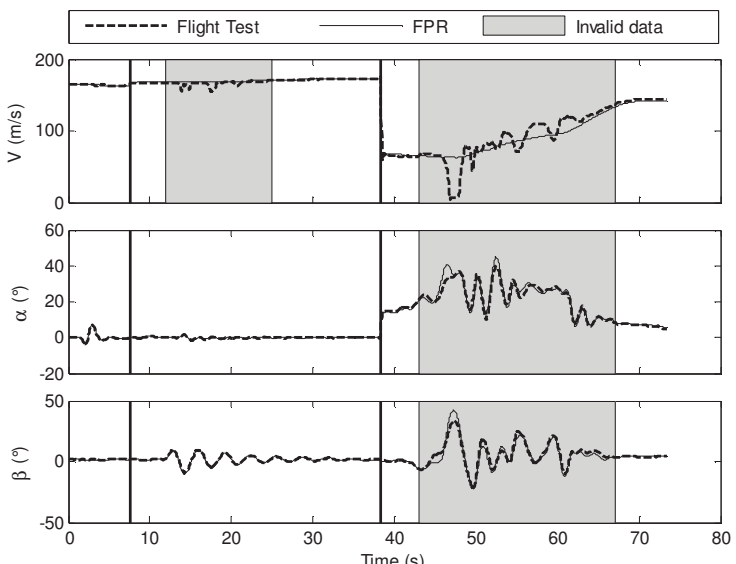

Fig. 8: FPR results for airspeed and flow angles (AT26 Xavante).

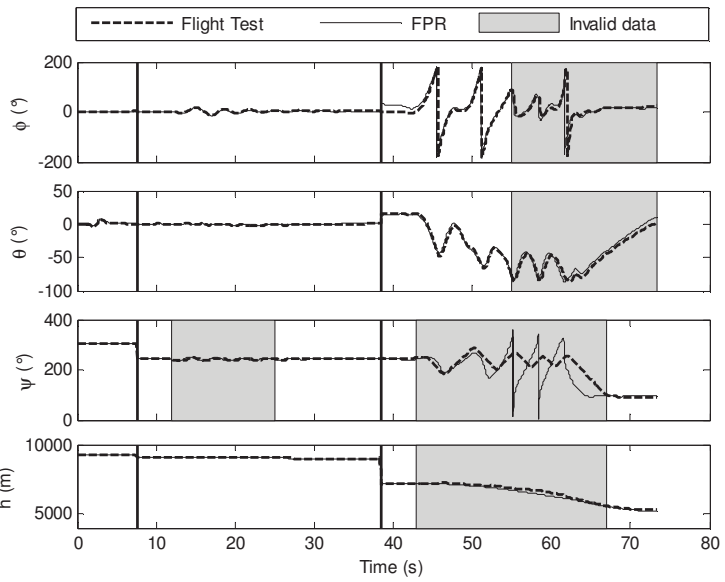

Fig. 9: FPR results for Euler angles and altitude (AT26 Xavante).

\section{Conclusion}

In this work, FPR was applied to spin tests performed with three different aircraft, featuring different FTI projects.

Despite the different levels of FTI limitations experienced, the flight path reconstruction proved to be capable of obtaining good estimates for the aircraft states during the spins.

This methodology also provided calibration for airflow angle vanes and the values of biases that affected the accelerometers and gyros in all cases analyzed.

\section{Acknowledgement}

The author gratefully acknowledges the support of IPEV (Instituto de Pesquisas e Ensaios em Voo) through flight test instrumentation project and test vehicle operation, and FINEP under agreement 01.13.0518.00 for financial support.

\section{References}

[1] Mulder, J. A., Chu, Q. P., Sridhar, J. K., Breeman, J. H. and Laban, M., "Non-linear Aircraft Flight Path Reconstruction Review and New Advances". Progress in Aerospace Sciences, No. 35, 1999, pp. 673-726.

[2] Jategaonkar, R. Flight Vehicle System Identification: a Time Domain Methodology, AIAA, Reston, Virginia, 2006.

[3] Stagg, G. A. An Unsteady Aerodynamic Model for Use in the High Angle of Attack Regime. Master's thesis, Virginia Polytechnic Institute and State University, Blacksburg, Virginia, 1998.

[4] Lewis, F. L.; Stevens, B. L. Aircraft Control and Simulation, $2^{\mathrm{a}}$ ed. Hoboken, New Jersey: John Wiley \& Sons, 2003.

[5] Klein, V. and Morelli, E. A., Aircraft System Identification, Theory and Practice, AIAA Education Series, 2006. 